\title{
Spatial Rules for Capturing Qualitatively Equivalent Configurations in Sketch maps
}

\author{
Sahib Jan, Carl Schultz, Angela Schwering and Malumbo Chipofya \\ Institute for Geoinformatics \\ University of Münster, Germany \\ Email: sahib.jan I schultzc I schwering I mchipofyal@uni-muenster.de
}

\begin{abstract}
Sketch maps are an externalization of an individual's mental images of an environment. The information represented in sketch maps is schematized, distorted, generalized, and thus processing spatial information in sketch maps requires plausible representations based on human cognition. Typically only qualitative relations between spatial objects are preserved in sketch maps, and therefore processing spatial information on a qualitative level has been suggested. This study extends our previous work on qualitative representations and alignment of sketch maps. In this study, we define a set of spatial relations using the declarative spatial reasoning system $\operatorname{CLP}(Q S)$ as an approach to formalizing key spatial aspects that are preserved in sketch maps. Unlike geo-referenced maps, sketch maps do not have a single, global reference frame. Rather, the sketched elements themselves act as referencing objects. Using the declarative spatial reasoning system $\operatorname{CLP}(Q S)$, we define constraint logic programming rules that formalize various key spatial aspects of sketch maps at a local level, between nearby objects. These rules focus on linear ordering, cyclic ordering, and relative orientation of depicted objects along, and around, salient reference objects.
\end{abstract}

\section{INTRODUCTION}

$\mathbf{S}$ KETCH maps are used to externalize an individual's mental image of the environment. The information represented in sketch maps is based on observation rather than measurements. Therefore, information in sketch maps is schematized, distorted, and generalized. Freehand sketch maps contain objects and spatial relations between these objects which enable users to use sketch maps to communicate and reason about actions in environments. During the last two decades, several approaches $[7,9,21,32]$ attempt to capture spatial configurations between depicted objects qualitatively. These approaches use different aspects of space such as topological relations, cardinal directions, relative orientations, and relative distances.

Throughout a series of experiments [27, 33, 34], Wang et al. identify a set of sketch aspects which are not subject to schematizations, distortions or any other cognitive impact. These sketch aspects represent: linear ordering, cyclic ordering, relative orientations, and topological relations between spatial objects. The identified sketch aspects are categorized into local and global levels [27]. The local level relations refer to the relations between nearby objects while global level relations represent possible relations between all objects in a map. During the last two decades, a series of qualitative spatial calculi have been proposed in the area of Qualitative Spatial Reasoning (QSR) [11] to formalize some of these aspects such as representations for the topological relations [6, 23], orderings $[1,22,25]$, directions $[10,24]$, relative position of points $[19,20,24]$ and others.

In our previous studies [14, 15, 16, 27], we propose a set of plausible representations and their coarse versions to qualitatively formalize key sketch aspects. We use several qualifiers to extract qualitative constraints from geometric representations of sketch and geo-referenced maps [13] in the form of Qualitative Constraint Networks (QCNs). QCNs are complete graphs representing spatial objects and relations between them. However, in order to derive more cognitively accurate QCNs, we require greater flexibility in being able to define qualitative spatial relations for our particular application domain, i.e. geographic-scale sketch maps. Specifically, sketch maps require qualitative constraints at a local level between particular types of adjacent objects such as linear ordering, cyclic ordering, and orientation information of nearby landmarks with respect to reference objects.

In this study, we propose the utilisation of the declarative spatial reasoning system $\operatorname{CLP}(\mathrm{QS})[2,26]$ as an alternative approach to deriving cognitively plausible constraints between nearby objects. The system is capable of modeling and reasoning about qualitative spatial relations within the context of the constraint logic programming. Using the CLP(QS) framework, we define logic programming rules over qualitative spatial domains in order to express and solve declarative, high-level constraints between spatial objects depicted in sketch maps.

The remainder of this paper is structured as follows: In the following section, we briefly introduce related work. In Section 3 we discuss spatial objects and cognitively plausible aspects found in sketch maps. In Section 4 we present CLP(QS) rules that formalize the cognitively salient aspects of sketch maps. Section 5 concludes the paper with an outlook on future work.

\section{RELATED WORK}

The information in sketch maps is based on observations rather than measurements. Therefore, processing spatial information on a qualitative level has been suggested [5, 27]. During the last two decades, several approaches [7, 9, 21, 32] attempt to capture spatial configurations between depicted objects qualitatively. Egenhofer et al. [8] propose SpatialQuery-by-Sketch, a sketch-based user interface that focuses on enabling a user to specify spatial relations (topology and 
cardinal directions) by drawing them. Volker et al. [31] propose the visual query system VISCO. It offers a sketch-based query language for defining approximate spatial constellations of the objects. Forbus et al. [9] develop a sketch understanding system CogSkech which is a space search system that focuses on topological relations among sketched elements, and reasons about these relations to infer new knowledge. Nedas et al. [21] propose a similarity measure methodology for comparing two spatial scenes by identifying cognitively-motivated similarities between objects, relations among spatial objects, and the ratio of the total number of objects in both scenes to the number of objects that have been correctly matched.

These approaches share our motivation of using abstract qualitative relations to represent spatial configurations between objects depicted by a user. In a previous study [27], we propose a framework to preprocess, align and integrate sketched spatial information on a qualitative level. The framework addresses the extraction of objects from sketch maps, computing QCNs from geometric representations of sketch and geo-referenced maps, and aligning them qualitatively.

This study extends our previous work on qualitative representations of spatial objects. As the outline of spatial objects in freehand sketches are imprecise, the qualitative representation of spatial objects with imprecise boundaries leads to different qualitative relations when compared to relations in geo-referenced maps. In this study, we present spatial rules defined using the CLP(QS) system as an alternative approach to compute qualitative relations (on a conceptual level) which are preserved in freehand sketch maps. These rules address linear ordering, cyclic ordering, and orientation information of adjacent objects along and around key reference objects.

\section{Spatial ObJects AND Their CONFIGURATIONS IN SKETCH MAPS}

\section{A. Spatial objects}

Inspired by the Lynch's seminal work [18] and Tversky's analysis of mental structures [30], we characterize the depicted objects in sketch maps into four elements: streets segments, junctions, landmarks, and city-blocks. These elements are automatically extracted using the object recognition method proposed in [4].

Street segments are connected, and mostly linear, features in sketch maps. They are represented as line segments and are connected to other street segments at junctions. The connectivity of street segments and the street-network is central for human path planning [12]. Junctions are the end-points of street segments. The end-points, where street segments are not connected to other street segments, are called hanging end-points. At the boundary of the sketching medium, street segments are left unconnected to any further street segment, resulting in hanging end-points. In sketch maps, junctions capture the connectivity of various street segments forming a street network. In our approach, both hanging end-points and junctions are spatially represented as 2D points.

Landmarks are the most salient elements in an environment and are therefore essential to characterize an environment. In freehand sketches, landmarks are vectorized and approximated by polygons which represent spatial entities such as water bodies, buildings, and parks. Landmarks and road entities are the most frequently depicted spatial objects in sketch maps [3], while city-blocks are the smallest regions. They are delimited by a lineal representation of connected street segments. People do not always sketch complete city-blocks, in particular at the edge of the sketch medium. Therefore, we define city-blocks as areas either bounded by the street segments, or bounded by street segments and the boundary of the medium [14].

\section{B. Invariant spatial aspects in Sketch Maps}

Processing sketch information at a qualitative level requires explicit knowledge about certain aspects of sketches that are not subject to schematizations, distortions, or any other cognitive impact [29]. That is, these aspects are preserved in freehand sketches. Throughout a series of experiments [27, 33, 34], Wang et al. identified a set of seven invariant sketch aspects. These aspects consist of: linear ordering of landmarks and street segments along a route, cyclic ordering of landmarks and street segments around reference junctions, relative orientation of landmarks with respect to street segments, topological and orientation relations between street segments in street network, and topological relations between extended objects (landmarks, and city-blocks). This paper focuses on the formalization of ordering and relative orientation of adjacent objects using spatial rules defined in the context of the CLP(QS) system.

\section{Spatial Rules For Qualitatively Equivalent CONFIGURATIONS}

Using the CLP(QS) framework, we define spatial rules to compute qualitative information between nearby objects. For the linear ordering and orientation information of adjacent landmarks, we use connected street segments as reference objects, while junctions are used as reference objects for cyclic ordering. The adjacency of landmarks is defined via relative metric distances.

\section{A. Preliminaries}

CLP(QS) includes a library of qualitative spatial relations encoded as polynomial constraints over a set of real variables $X$, which are solved via constraint logic programming [2]. In this subsection we present the CLP(QS) library implementations of projection, distance, and orientation relations that we build on in subsequent sections. A set of spatial relations is consistent in CLP(QS) if there exists some assignment of reals to the variables $X$ such that all of the corresponding polynomial constraints are satisfied. CLP(QS) uses a variety of polynomial solvers including CLP(R), SAT Modulo Theories, quantifier elimination by Cylindrical Algebraic Decomposition, and geometric constraint solvers.

Projection. A point is projected onto a line using the dot product. This is extended to segment-line projection by projecting both end points. Polygons are projected onto lines by projecting all vertices and taking the maximum and minimum 
projected values as the projected interval. Points are projected onto segments by clamping the projected value to lie within the projection of the segment and the line collinear with the segment, i.e. let $v, a, b$ be reals such that $a \leq b$ then

$$
\operatorname{CLAMP}(v, a, b)= \begin{cases}v & \text { if } a \leq v \leq b \\ a & \text { if } v<a \\ b & \text { if } v>b\end{cases}
$$

$\mathrm{CLP}(\mathrm{QS})$ projection predicates are implemented as follows.

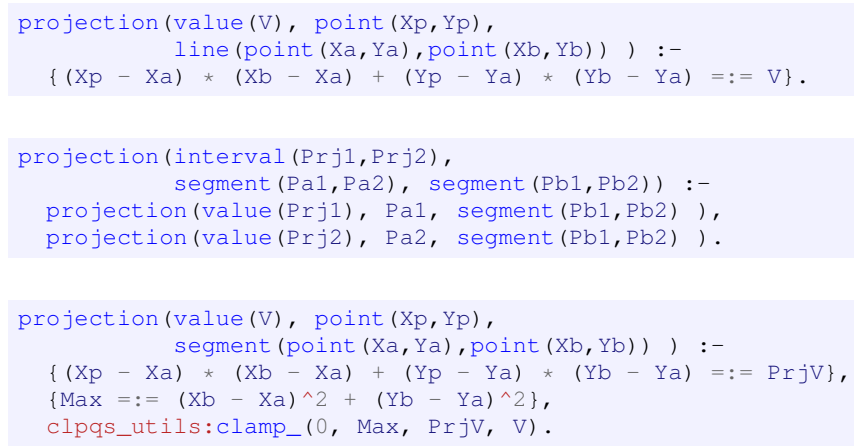

The CLP(QS) predicate for obtaining the coordinates of the projection onto a segment (or line) is:

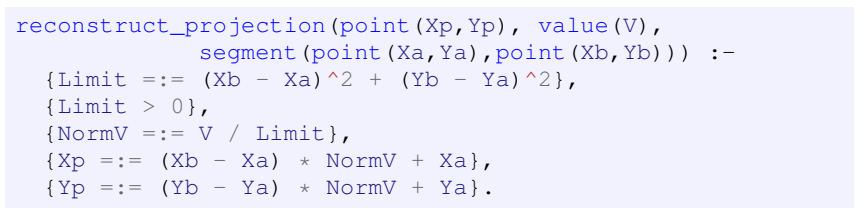

Euclidean distance. We employ CLP(QS) Euclidean distances between points, and between points and segments.

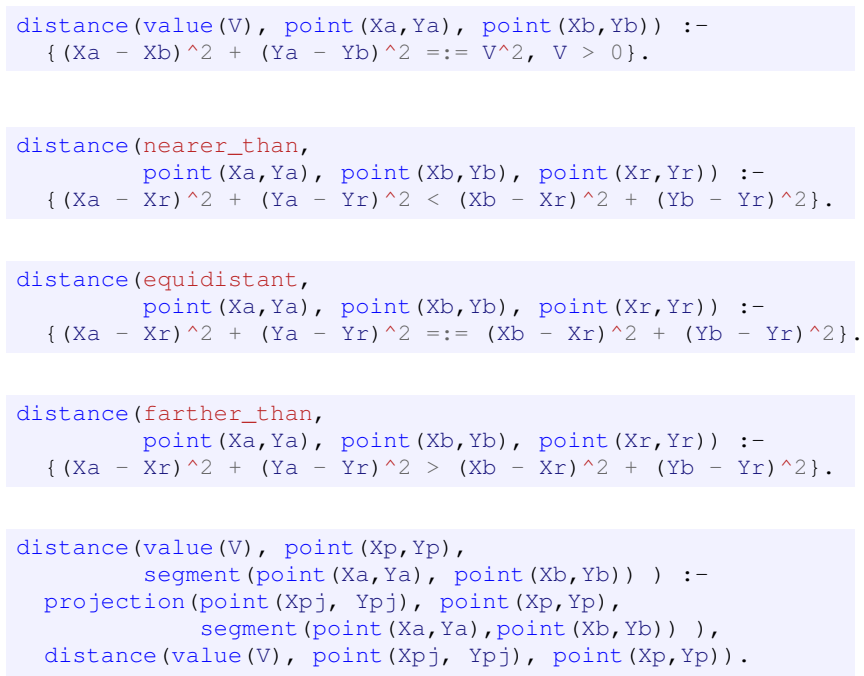

Relative Orientation. We employ $\mathrm{CLP}(\mathrm{QS})$ relative orientation predicates between points and lines.

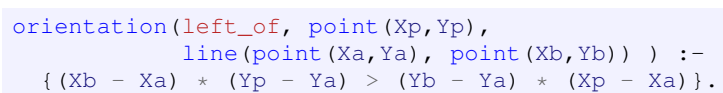

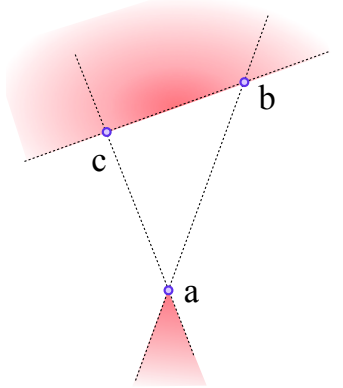

Fig. 1. Inconsistent relative orientation relations between triangle edges $A, B, C$ and a point $D$ : the point $D$ must occupy the intersection of the red regions. As the red regions are disconnected, the constraints are unsatisfiable.

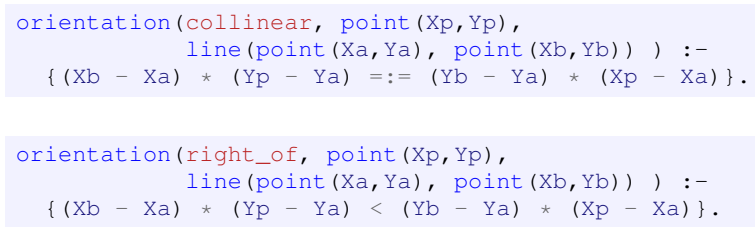

Spatial reasoning with incomplete numerical information. We emphasise that, using $\operatorname{CLP}(\mathrm{QS})$, it is possible to reason about sets of spatial relations in the partial or complete absence of numerical information. For example, we can define an anticlockwise triangle with vertices $A, B, C$ with the constraint that $C$ is on the left of line $(A, B)$. A point $D$ can then be constrained by relative orientation relations with the triangle edges:

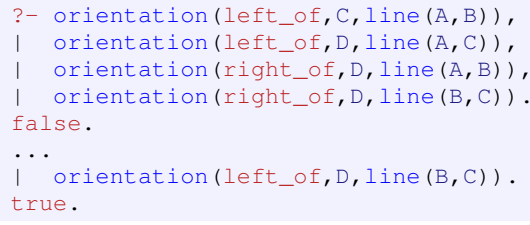

Even though we have not provided any numerical information about the positions of the points $A, B, C, D, \operatorname{CLP}(\mathrm{QS})$ correctly determines that $D$ cannot be simultaneously left of $(A, C)$ and right of $(A, B)$ and $(B, C)$ as illustrated in Figure 1. We use this feature to reason about possible scenarios in sketch maps in cases where only incomplete numerical information is available.

\section{B. Rules for Linear Ordering as Constraints}

In sketch maps, linear ordering of spatial objects is an invariant sketch aspect [33]. It describes the linear ordering of adjacent landmarks and street segments along a route. A route is defined as a set of connected street segments. In [33, 34] Wang et al. found the linear ordering of both landmarks and street segments as a suitable representation for sketch map alignment. In our previous studies [16], we proposed a coarse version of Allen's interval algebra [1] to extract the linear ordering of spatial objects along a route.

We define spatial rules to compute the linear ordering of adjacent landmarks and connected street segments along a 


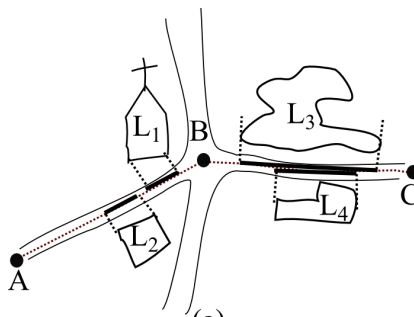

(a)
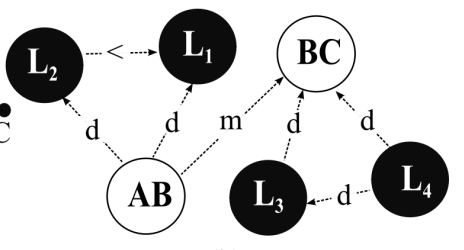

(b)
Fig. 2. (a) Spatial objects in the sketch map (b) Ordering of adjacent landmarks and connecting street segments along the route $(\mathrm{AB}, \mathrm{BC})$

route. The adjacency of landmarks is computed using the relative metric distance between street segments and landmarks. A landmark is considered adjacent or local if its footprint intersects with the buffer around the reference street segment. Next, adjacent landmarks are projected from both sides of a route onto the street segments. As proposed in our previous studies [16], the ordering between intervals of projected landmarks and street segments are represented using both Allen's Interval relations and a coarse version of Allen's interval relations [1]. This is accomplished by encoding qualitative spatial relations as rules and facts using $\operatorname{CLP}(\mathrm{QS})$, as illustrated in Figure 2. Figure $2 \mathrm{~b}$ shows the linear ordering of the depicted objects in terms of Allent's relations such as meets $(\mathrm{m})$, before $(<)$, and during(d).

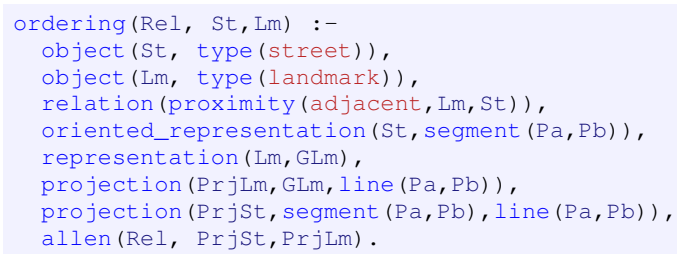

Buffer Size. In sketch maps, the distances between depicted objects are distorted. The distances between landmarks and adjacent street segments are always variant between sketch maps of the same location. Therefore, defining a common buffer size for all freehand sketch maps is not possible. In order to compute relative buffer, we define an automatic method as rule in $\operatorname{CLP}(\mathrm{QS})$ which computes minimum distances between landmarks and street segments and then considers the maximum distance as buffer size from the computed minimum distances.

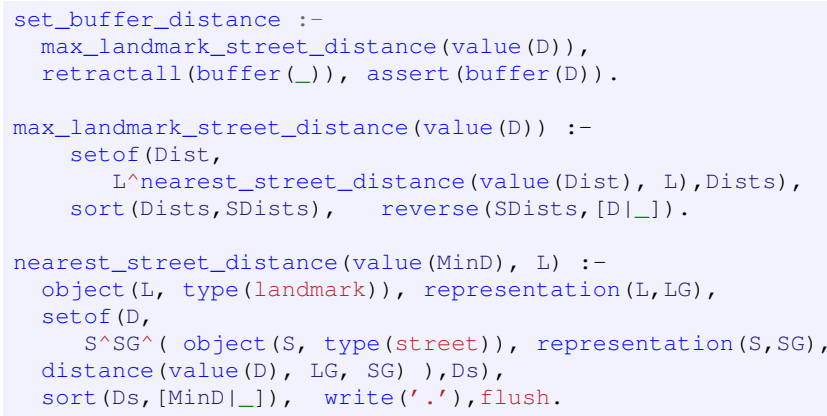

Adjacency. We define a rule for adjacency as a qualitative constraint between landmarks and street segments. A landmark is considered adjacent when the polygonal footprint of the landmark intersects with the buffer of the reference street segment. The adjacency rule is used to define linear ordering locally, between nearby objects.

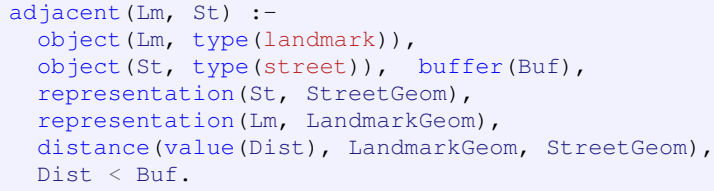

Linear Projection. This spatial rule projects landmarks onto adjacent street segments. The projections of landmarks onto street segments are represented as pairs of intervals with start and end points. The rule also projects spatial entities such as point-to-line, line-to-line, and polygon-to-line objects. The projected intervals together with the intervals of street segments provide ordering information between spatial objects along a route.

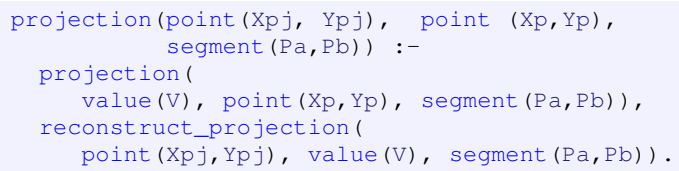

Qualitative Interval Ordering. These spatial rules define linear ordering relations between projected intervals. The rule compares the start and end points of two intervals and represents them as relations as defined in Allen's Interval Algebra (IA).

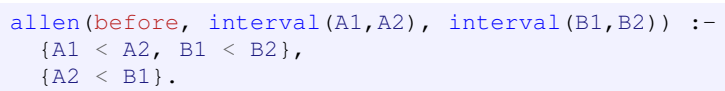




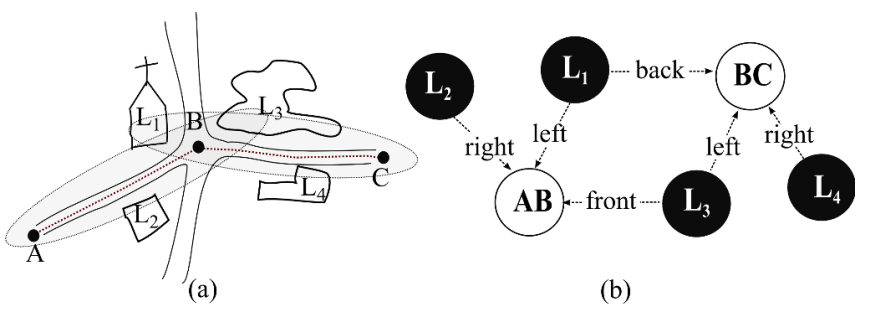

Fig. 3. (a) Orientation of adjacent landmarks with respect to street segments (b) Qualitative constraints representing orientation relations.

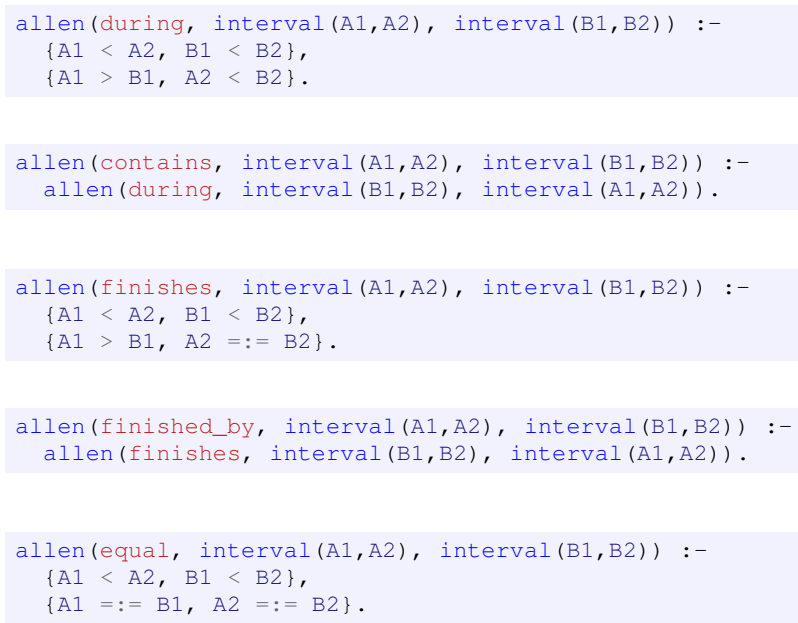

\section{Rules for Relative Orientation of Landmarks}

It is common to use points as basic entities in positional reasoning [11, 19]. In [14], we investigate different qualitative representations in order to formalize orientation information of landmarks with respect to adjacent street segments and relations defined in the Left-Right (LR) calculus [28]. The LR calculus deals with point type entities in the plane $R^{2}$. It describes the position of a point $C$ with respect to two other points $A$ (the origin) and $B$ (the relatum). However, in sketch maps landmarks are extended objects approximated by polygons and considering the centroids of landmarks loses shape information of the depicted objects.

We propose the following relations for representing relative orientation of landmarks and street segments at a conceptual level in sketch maps (see Figure 3). Our representation consists of six binary relations between two objects: left_of, right_of, crosses, crossed_by, front_of, and back_of. These relations capture key equivalence classes of spatial configurations that are preserved in sketch maps.

Orientation Relations. These rules define six orientation relations between depicted landmarks and street segments. A landmark is considered to be on the left of a street segment if all of the vertices of the polygonal representation of the landmark are on the left side of the street segment. The landmark crosses an adjacent street segment if some vertex of the landmark is left_of the street segment, some vertex is right_of the street segment, and the projection of the landmark onto the line collinear with the street intersects the street segment.

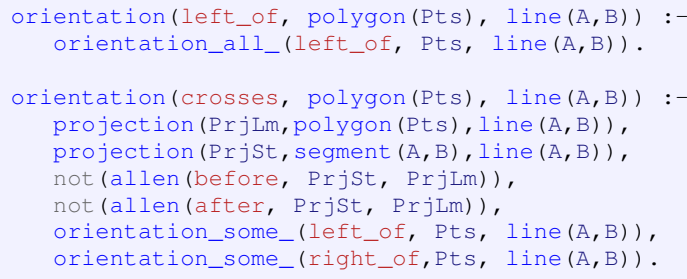

A landmark is in front_of an adjacent street segment if some vertex of the landmark is on the left_of the street segment, some vertex is on the right_of the street segment, and the projection of the landmark onto the line collinear with the street is not on the street segment (see Figure $3 b$ ). The respective inverse orientation relations are: right_of, crossed_by and back_of.

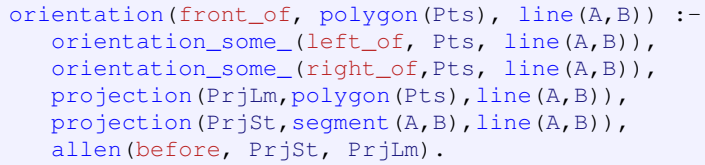

\section{Rules for Cyclic Ordering as Constraints}

This sketch aspect describes the angular ordering of nearby landmarks and connected street segments as referent objects around a street junction. Similar to linear ordering, Wang et al. [33, 34] find the cyclic ordering of both landmarks and street segments around reference junctions as a suitable representation for sketch map alignment.

In [16], we proposed a coarse version of the Cyclic Interval Algebra (CIA) [22] to formalize cyclic ordering of depicted sketch map objects around reference junctions. The c-intervals of landmarks and street segments are their projections onto a central point, i.e. a reference junction. The projection is given by sweeping the $360^{\circ}$ view at a junction in a counter-clockwise direction. We define spatial rules in CLP(QS) for deriving the cyclic ordering of depicted objects. As with linear ordering, the spatial rule is used to generate a qualitative constraint network between landmarks and street segments based on adjacency, cyclic projections, and qualitative relations between cyclic intervals.

Adjacency Buffer Size. Analogous to the computation of buffer size around a street segment, this rule defines the buffer size around a reference object. The buffer size around junctions is used to define adjacency relations between landmarks and reference junctions. 


\section{DisCUSSION AND USE CASE}

In this section we discuss preliminary results of using our representations for matching. We have conducted a simple pilot study to investigate the ways in which various representations can be combined and utilised for the task of matching (i.e. aligning) spatial objects from sketch maps with georeferenced maps.

The objective of the alignment task is to accurately match objects from the sketch map with objects in the geo-referenced map according to their relevant qualitative spatial relations. This is a challenging task in general [27], and we are investigating the use of a "mosaic" of different representations that can be combined to increase the accuracy of the matching process, e.g. a combination of topological relations between city blocks, linear representations between landmarks along street segments, cyclic representations between landmark around junctions, and so on.

In this simple pilot study we explore the combination of topological relations between city blocks and linear relations between landmarks based on a real, user-generated sketch map of Münster, Germany (see Figures 6 and 7). That is, we firstly match the maps based on city blocks using topological relations (primarily adjacency), as reported in [17]. As we report in [17], city blocks can often be matched accurately based on topological information. Figure 8 illustrates the extract of the sketch map (Figure 6) and geo-referenced map (Figure 7) comparing landmarks within a correctly matched city block.

We employ our linear representation to match landmarks within each city block. Landmarks are linearly ordered based on their projection onto the streets that define the city block. Figure 8 illustrates the derived ordering of the landmarks; for clarity, only the upper horizontal street in the sketch map is shown. The light grey region indicates the proximity threshold used to define adjacency of landmarks with respect to street segments. Projection of the landmark is based on the region of the landmark within the proximity threshold. Based on these projections, landmarks $r_{2}, r_{3}, r_{4}$ maintain a similar ordering in both the sketch and geo-referenced maps, thus assisting in the matching between landmark objects within a city block.

This pilots study also highlights the complexity of the matching task: observe that landmark $r_{1}$ was not sketched by the participant (i.e. $r_{1}$ is missing from the sketch map, Figure 8(a)). Moreover, according to our projection representation in the geo-referenced map (Figure 8(b)), $r_{1}$ overlaps $r_{2}$ in the first street of the geo-referenced map, but is also projected onto the last street that defines the block. We need to formalise further spatial domain knowledge in order for a matching algorithm to correctly interpret such combinations of qualitative relations between landmarks along a path.

The evaluation of proposed representations based on qualitative matching of sketch maps with geo-referenced maps is ongoing research work. In future work we are also investigating alternative landmark projections. For example, the front face of the polygon may be better indication of how people perceive the ordering information of landmarks along a particular route,

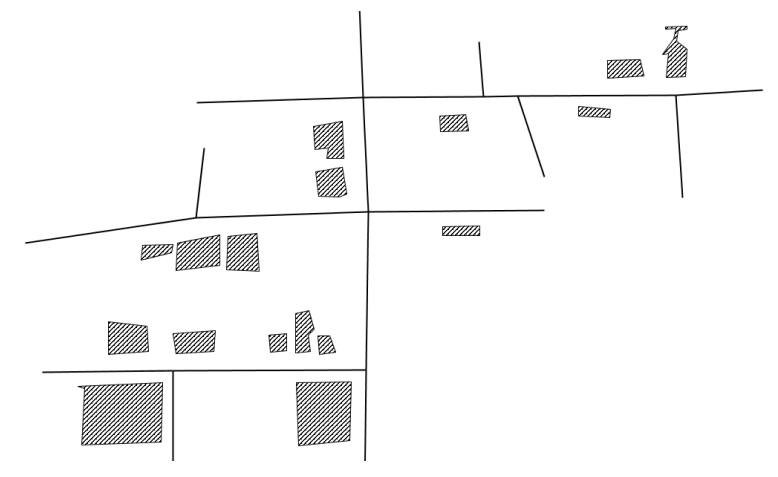

Fig. 6. Sketch map of a region in Münster, Germany.

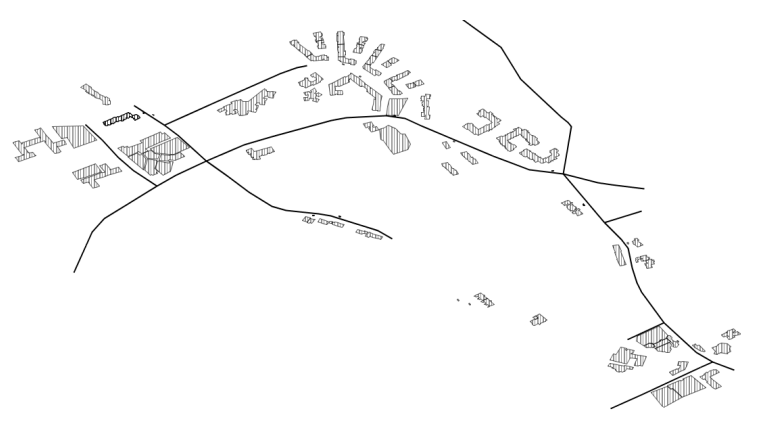

Fig. 7. Geo-referenced map of Münster, Germany.

rather than the projection of the entire polygon within the proximity threshold.

\section{CONCLUSIONS}

In this paper we introduce a set of spatial relations that formalize key equivalence classes of spatial configurations preserved in sketch maps; the relations have been defined using the declarative spatial reasoning system CLP(QS). Our rules address: (1) the linear ordering of landmarks and street segments along any route; (2) the cyclic ordering of landmarks and connected street segments around reference junctions; (3) the orientation of landmarks with respect to nearby street segments. The rules also define an appropriate buffer size in sketch maps using the minimum distances between landmarks and street segments. The buffer size is used to define proximity and adjacency relations as qualitative relations between nearby objects.

We derive ordering and relative orientation relations as sets of Prolog facts using CLP(QS). We then use these facts to generate Qualitative Constraint Networks (QCNs) of sketch and geo-referenced maps. These qualitative networks are used in a range of tasks, for example (a) to provide a high-level qualitative query interface to both sketched and geo-referenced maps, and (b) to facilitate matching sketched maps to georeferenced maps i.e. aligning qualitative spatial information in sketch maps with corresponding spatial information in georeferenced maps. The evaluation of proposed representations based on qualitative matching of sketch maps with georeferenced maps is ongoing research work. 


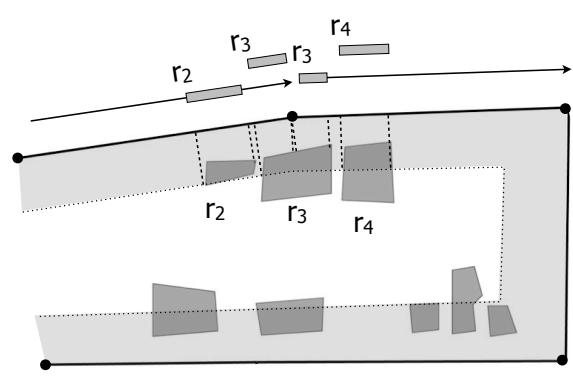

(a) Sketch map

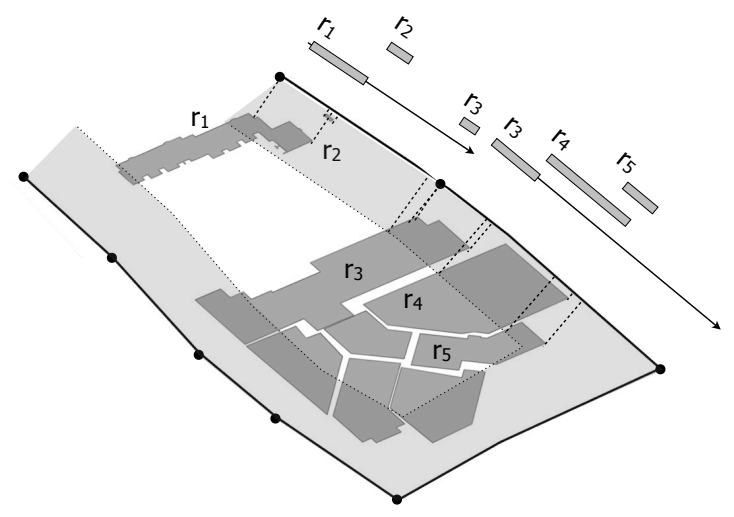

(b) Geo-referenced map

Fig. 8. Deriving linear ordering relations of projected landmarks per block in the sketch and geo-referenced map; only the projection of adjacent landmarks onto the upper horizontal street is illustrated.

\section{ACKNOWLEDGMENT}

This work is funded by the German Research Foundation (DFG) under grant for a SketchMapia project (Grant SCHW 1372/7-1).

\section{REFERENCES}

[1] J. F. Allen. Maintaining Knowledge about Temporal Intervals. Communications of the ACM, 26(11):832-843, 1983.

[2] M. Bhatt, J. H. Lee, and C. Schultz. CLP(QS): A Declarative Spatial Reasoning Framework. In proceedings of the 10th international conference on Spatial information theory (COSIT-11), pages 210-230, Berlin Heidelberg, 2011. SpringerVerlag.

[3] A. Blaser and M. J. Egenhofer. A visual tool for querying geographic databases. In Proceedings of the working conference on Advanced visual interfaces, number 1, pages 211-216, New York, New York, USA, 2000. ACM. ISBN 1581132522. doi: 10.1145/345513.345318

[4] K. Broelemann. A System for Automatic Localization and Recognition of Sketch Map Objects. In Workshop of Understanding and Processing Sketch Maps, pages 11-20, Belfast, Maine, 2011. AKA Verlag.

[5] M. Chipofya, J. Wang, and A. Schwering. Towards cognitively plausible spatia representations for sketch map alignment. In M. Egenhofer, N. Giudice, R. Moratz M.Worboys (eds.) Spatial Information Theory, LNCS , Vol .6899, pages 20-39. Springer, Berlin Heidelberg, 2011

[6] A. G. Cohn. Qualitative Spatial Representation and Reasoning Techniques. In Advances in Artificial Intelligence 1997. LNAI, Vol. 1303, pages 1-30. Springer Berlin Heidelberg, 1997.

[7] M. J. Egenhofer. Spatial-Query-by-Sketch. In M. Burnett and W. Citrin (eds.), IEEE Symposium on Visual Languages (IEEE-96), Vol. 96, pages 60-67. IEEE Press, Boulder, Colorado, 1996

[8] M. J. Egenhofer. Query processing in spatial-query-by-sketch. In the Journal of Visual Languages and Computing, 8(4):403-424, 1997.

[9] K. Forbus, J. Usher, A. Lovett, K. Lockwood, and J. Wetzel. CogSketch: Opendomain sketch understanding for cognitive science research and for education. volume 4, pages 648-666, 2011. doi: 10.1111/j.1756-8765.2011.01149.x.

[10] A. U. Frank. Qualitative spatial reasoning: cardinal directions as an example. In International journal of geographical information systems, 10(3):269-290, Apr 1996. ISSN 0269-3798. doi: 10.1080/02693799608902079.
[11] C. Freksa. Dimensions of qualitative spatial reasoning. In In N.P. Carreté, M.G. Singh (eds.) Proceeding III MACS-International Workshop on Qualitative Reasoning and Decision Technologies QUARDET'93, number June 1993, pages 483-492. CIMNE, Barcelona, 1993.

[12] N. T. Huynh and S. T. Doherty. Digital Sketch-Map Drawing as an Instrument to Collect Data about Spatial Cognition. Cartographica: The International Journal for Geographic Information and Geo-visualization, 42(4):285-296, Jan. 2007. ISSN 0317-7173. doi: 10.3138/carto.42.4.285.

[13] S. Jan and M. Chipofya. Integration of Qualitative Spatial Reasoning into GIS An Example with SparQ. In Schwering, A. Pebesma, E. Behncke, K. (eds.), In Geoinformatik 2011, pages 63-78, Münster, Germany., 2011.

[14] S. Jan, A. Schwering, M. Chipofya, and T. Binor. Qualitative Representations of Extended Spatial Objects in Sketch Maps. In J. Huerta et al. (eds.), Connecting a Digital Europe Through Location and Place, LNG\&C, pages 37-54. Springe International Publishing, Switzerland, 2014. doi: 10.1007/978-3-319-03611-3\_3,

[15] S. Jan, A. Schwering, M. Chipofya, and J. Wang. Qualitative Representations of Schematized and Distorted Street Segments in Sketch Maps. In International Conference, Spatial Cognition IX, LNCS, Vol. 8684, pages 253-267, Bremen, Germany, 2014. Springer International. URL http://ifgi.uni-muenster.de/ s $\backslash$ jan001/ Publications/sc2014.pdf.

[16] S. Jan, A. Schwering, J. Wang, and M. Chipofya. Ordering: A Reliable Qualitative Information for the Alignment of Sketch and Metric Maps. In International Journal of Cognitive Informatics and Natural Intelligence (IJCINI-2014), 8(1):68-79, 2014.

[17] S. Jan, A. Schwering, C. Schultz, and M. Chipofya. RCC11: A formal topological representation for the alignment of Extended Objects in Sketch Maps. In 28th International Workshop on Qualitative Reasoning (QR-2015), August 10-12, Minneapolis, MN, USA, 2015.

[18] K. Lynch. The image of the city. Cambridge MA,USA: MIT Press, 1960. ISBN 0262620014

[19] R. Moratz, J. Renz, and D. Wolter. Qualitative spatial reasoning about line segments. In Proceedings of the 14th European Conference on Artificial Intelligence (ECAI 2000), pages 234-238. IOS Press, Amsterdam, 2000.

[20] R. Moratz, F. Dylla, and L. Frommberger. A Relative Orientation Algebra with Adjustable Granularity. In proceedings of the Workshop on Agents in Real-time and Dynamic Environments (IJCAIO5), Edinburgh, Scotland, UK, 2005.

[21] K. A. Nedas and M. J. Egenhofer. Spatial-Scene Similarity Queries. In Transactions in GIS, 12(6):661-681, 2008

[22] A. Osmani. Introduction to Reasoning about Cyclic Intervals. In In Imam I. Kodratoff, Y., El-Dessouki, A and Ali, M. (Eds.) Multiple Approaches to Intelligent Systems. In Lecture Notes in Computer Science, Vol. 1611, Springer Berlin-Heidelberg, volume 1611, pages 698-706, 1999.

[23] D. A. Randell, Z. Cui, and A. G. Cohn. A Spatial Logic based on Regions and Connection. In B. Nebel, C. Rich, and W. R. Swartout (Eds.), In Proceeding of the 3rd National Conference on Principles of Knowledge Representation and Reasoning, Cambridge, MA, 1992. Morgan Kaufmann Publishers Inc.

[24] J. Renz and D. Mitra. Qualitative Direction Calculi with Arbitrary Granularity. In C. Zhang, H. Guesgen, W.Yeap (eds.), PRICAI-04: Trends in Artificial Intelligence, LNCS, Vol. 3157, pages 65-74. Springer, Berlin Heidelberg, Auckland, New Zealand, 2004. doi: 10.1007/978-3-540-28633-2\9.

[25] C. Schlieder. Reasoning about ordering. In Andrew U. Frank, Werner Kuhn (Eds.): Spatial Information Theory: A Theoretical Basis for GIS, International Conference COSIT '95, Semmering, Austria, September 21-23, 1995.

[26] C. Schultz and B. Mehul. Towards a Declarative Spatial Reasoning System. In proceedings of the 20th European Conference on Artificial Intelligence (ECAI-12), Montpellier, France, 2012. ISO Press.

[27] A. Schwering, J. Wang, M. Chipofya, S. Jan, R. Li, and K. Broelemann. SketchMapia: Qualitative Representations for the Alignment of Sketch and Metric Maps. Spatial Cognition \& Computation: An Interdisciplinary Journal, 14(3):220254, July 2014. ISSN 1387-5868. doi: 10.1080/13875868.2014.917378. URL http://www.tandfonline.com/doi/abs/10.1080/13875868.2014.917378.

[28] A. Scivos and B. Nebel. The Finest of its Class: The Natural, Point-Based Ternary Calculus LR for Qualitative Spatial Reasoning. In C. Freksa et al. (2005), Spatial Cognition IV. Reasoning, Action, Interaction: International Conference Spatial Cognition. Lecture Notes in Computer Science Vol. 3343, Springer, Berlin Heidelberg, volume 3343, pages 283-303, 2004

[29] B. Tversky. Distortions in cognitive maps. In Geoforum-interdisciplinary Journal, 23(2):131-138, May 1992. ISSN 00167185 doi: 10.1016/0016-7185(92)90011-R.

[30] B. Tversky. Structures of Mental Spaces: How People Think About Space. Environment \& Behavior, 35(1):66-80, 2003.

[31] H. Volker and W. Michael. Querying GIS with Animated Spatial Sketches. In Proceeding IEEE Symposium on Visual Languages, pages 197-204, Isle of Capri, Italy, 1997. IEEE Press.

[32] J. Wallgrün, D. Wolter, and K.-F. Richter. Qualitative Matching of Spatial Information. In proceedings of the 8th SIGSPATIAL International Conference on Advances in Geographic Information Systems, pages 300-309, USA, 2010. ACM New York. ISBN 9781450304283.

[33] J. Wang and A. Schwering. Invariant spatial information in sketch maps: Towards a sketching interface for collaborative mapping. In Journal of Spatial Information Science, 2014.

[34] J. Wang, C. Mülligann, and A. Schwering. A Study on Empirically Relevant Aspects for Qualitative Alignment of Sketch Maps. In proceedings of the Sixth international conference on Geographic Information Science (GIScience), 2010. 